\title{
The functional polymorphism rs73598374:G $>A$ (p.Asp8Asn) of the ADA gene is associated with telomerase activity and leukocyte telomere length
}

\author{
Fabio Concetti ${ }^{1}$, Francesco M Carpi ${ }^{2}$, Massimo Nabissi ${ }^{3}$, Matteo Picciolini ${ }^{4}$, Giorgio Santoni ${ }^{3}$ \\ and Valerio Napolioni ${ }^{\star, 4}$
}

Recent evidence demonstrated a relevant role of adenosine deaminase (ADA) in replicative senescence of T cells through its capacity to modulate telomerase activity (TA). Herein, we tested the impact of the functional polymorphism ADA rs73598374:G $>$ A (c.22G $>$ A, p.Asp8Asn) on telomere biology, by measuring TA and leukocyte telomere length (LTL) in healthy subjects selected according to rs73598374 genotype. rs73598374-A carriers showed lower TA $(P=0.019)$ and shorter LTL $(P=0.003)$, respectively, compared to $\mathrm{G} / \mathrm{G}$ carriers. rs73598374-A carriers showed a stronger cross-sectional age reduction of LTL $(r=-0.314, P=0.005)$ compared to $G / G$ carriers $(r=-0.243, P=0.022)$. The reduced ADA activity associated to rs73598374-A variant predisposes those carriers to display higher levels of adenosine compared to $G / G$ carriers. Consequently, it may lead to an accelerated process of replicative senescence, causing a stronger reduction of TA and in turn shorter LTL. In conclusion, the crucial role played by replicative senescence of the immune system in several human diseases and in the aging process underscores the relevance of the present findings and also spurs interest into the possible involvement of rs73598374 in shaping the susceptibility to several age-related diseases.

European Journal of Human Genetics (2015) 23, 267-270; doi:10.1038/ejhg.2014.102; published online 4 June 2014

\section{INTRODUCTION}

Adenosine deaminase (ADA; EC3.5.4.4), encoded by ADA gene (gene map locus 20q13.11), catalyzes the hydrolytic deamination of adenosine or $2^{\prime}$-deoxyadenosine to inosine or $2^{\prime}$-deoxyinosine and ammonia. Congenital defect of ADA causes severe combined immunodeficiency, which is characterized by the absence of functional $\mathrm{T}$ and $\mathrm{B}$ cells in affected individuals. ${ }^{1} \mathrm{ADA}$, present both intracellularly and on the cell surface as ecto-ADA bound to CD26, ${ }^{1}$ is essential for optimal function of mature $\mathrm{T}$ cells. Indeed, the ADA/CD26 complex, which is a component of the immunological synapse, delivers a co-stimulatory signal upon T-cell stimulation..$^{1,2}$ Moreover, ADA, by regulating adenosine (that is a well-known immunosuppressive agent) levels, influences multiple T-cell functions, including IL-2 production, ${ }^{3}$ but also affects the replicative senescence of $\mathrm{T}$ cells and the expression of $\mathrm{CD} 28$ in cell culture. ${ }^{4}$ In particular, it has been demonstrated that $\mathrm{CD}^{+}{ }^{+} \mathrm{CD} 28^{+} \mathrm{T}$ cells lacking $\mathrm{ADA}$ surface expression display significantly less telomerase activity (TA) than those expressing $\mathrm{ADA}$, and that $\mathrm{ADA}$ surface receptor expression continuously declines during the progression of $\mathrm{T}$ cells to senescence in cell culture. ${ }^{4}$ Notably, a recent study further underscores the impact of ADA on replicative senescence by showing a significant correlation of $\mathrm{CD}_{8}{ }^{+} \mathrm{T}$-cell $\mathrm{TA}$ with the percentage of $\mathrm{CD}^{+}{ }^{+} \mathrm{T}$ cells that were $\mathrm{ADA}^{+}$in $\mathrm{HIV}-1^{+}$individuals. ${ }^{5}$

The ADA gene displays a $\mathrm{G}>\mathrm{A}$ single-nucleotide polymorphism (SNP, rs73598374, NM_000022.2:c.22G > A) at nucleotide 22 of exon 1 leading to an Asp (allele $1^{*}$ ) to Asn (allele $2^{*}$ ) amino-acid substitution at position 8 of the mature protein (NP_000013.2:p. Asp8Asn) that influences ADA activity. ADA rs73598374-A displays an $\sim 35 \%$ lower activity than that of the $A D A$ rs73598374-G and, consequently, carriers of $A D A$ rs73598374-A show a higher level of both circulating and intracellular adenosine. ${ }^{6,7}$ The frequency of the $A D A$ rs73598374-A allele is estimated to be 0.07 in Western populations, but it is lower among individuals of African and Asian descent (http://www.1000genomes.org/). We previously reported a significant association of $A D A$ rs73598374 with human life expectancy. ${ }^{8,9}$ Herein, we tested the impact of $A D A$ rs73598374 SNP on telomere biology, by measuring TA and leukocyte telomere length (LTL in healthy subjects selected according to rs73598374 genotype.

\section{MATERIALS AND METHODS}

Subjects

Studied samples were selected from a healthy Italian-Caucasian population of 1072 subjects genotyped for $A D A$ rs73598374:G > A (NM_000022.2:c.22G > A) (http://genomics.senescence.info/longevity/entry.php?id=1393). ${ }^{9}$ For TA, peripheral blood mononuclear cells (PBMCs) were isolated from 20 subjects (mean age $27.8 \pm 1.4$ years) matched for age, sex and rs73598374 genotype. One hundred sixty-eight samples (mean age $71.8 \pm 23.5$ years, females $N=86$, males $N=82$, rs73598374 G/G $N=89, \quad \mathrm{~A}+=79$ ) were used to determine LTL (www.gwascentral.org/study/HGVST1715). The study protocol was approved by the Joint Ethical Committee (JEC) University of Camerino-Azienda ASUR Marche ZT-10 Camerino, in accordance with the Declaration of Helsinki in its revised edition and with international and local regulatory requirements.

${ }^{1}$ IRCCS Neuromed, Pozzilli, Italy; ${ }^{2}$ School of Biosciences and Biotechnologies, University of Camerino, Camerino, Italy; ${ }^{3}$ School of Pharmacy, University of Camerino, Camerino, Italy; ${ }^{4}$ Innovation Pole for Genomics, Genetics and Biology, Perugia, Italy

${ }^{*}$ Correspondence: Dr V Napolioni, Innovation Pole for Genomics, Genetics and Biology, Via Gambuli, Building D, 3rd Floor, 06132 Perugia, Italy. Tel: + 393334307577 ; Fax: + 39 0737402007; E-mail: napvale@gmail.com

Received 2 September 2013; revised 18 March 2014; accepted 30 April 2014; published online 4 June 2014 


\section{TA assay}

TA was quantified with TeloExpress Quantitative Telomerase Detection Kit (XpressBio, Thurmont, MD, USA), which utilizes the telomerase repeat amplification protocol-based qPCR method, according to the manufacturer's instruction. TA was expressed as attomoles of DNA product by $1 \mathrm{ng}$ of protein lysate (quantified by Bradford assay) in a minute (attomoles/ng $\times$ min), and it was calculated based on the threshold cycle $(\mathrm{Ct})$ compared with the standard curve generated from 10-fold dilutions of telomerase control oligo with known copy numbers of the telomeric repeats.

\section{LTL measurement}

LTL was assessed by monochrome multiplex Real-Time qPCR, by calculating the $\mathrm{T} / \mathrm{S}$ ratio, which is a relative measure of telomere length expressed in arbitrary units for each sample by using a slightly modified version of the 2009 Cawthon method. ${ }^{10,11}$ Briefly, a total $25 \mu \mathrm{l}$ of PCR reaction mix containing 20 ng genomic DNA, SYBR Green JumpStart Taq ReadyMix 2x, 1 m betaine, $1 \mathrm{~mm}$ DTT, $100 \mathrm{~nm}$ telg, $900 \mathrm{~nm}$ telc, $700 \mathrm{~nm}$ albugcr2, and $500 \mathrm{~nm}$ albdgcr2 was prepared for each sample (all the primers and reagents were purchased from Sigma-Aldrich, St Louis, MO, USA). In order to calculate reliable T/S ratios, the samples were randomized by genotype, run in triplicate, and compared against a 5-point standard curve (made with 81-fold-range serial dilutions of a reference DNA sample), run in triplicate and in the same well position, for each plate. During the thermal cycle telomere amplification signal was collected at $74^{\circ} \mathrm{C}$ in the early cycles when albumin signal was under baseline, while acquisition at $85^{\circ} \mathrm{C}$ provided the albumin $\mathrm{Ct}$ values. The intra-assay coefficients of variation (CVs) were $2.1 \%$ for $\mathrm{T}, 2.2 \%$ for $\mathrm{S}$ and $4.8 \%$ for $\mathrm{T} / \mathrm{S}$ ratio. Three samples were repeatedly included in each plate (loaded in triplicate and in the same well position), in order to calculate the inter-run $\mathrm{CV}$ and rule out results from plates when this coefficient was bigger than 5\%. To check for PCR reaction specificity, a melting analysis step was included at the end of each PCR run. In our experimental conditions the standard curves' correlation coefficients $\left(R^{2}\right)$ were all $>0.99$.

\section{Statistical analysis}

The effect of ADA rs73598374 SNP on TA and LTL was assessed using the general linear model and stepwise linear regression. A genetic dominant model was used $\left(\mathrm{A}+v_{s} \mathrm{G} / \mathrm{G}\right)$ since only two $\mathrm{A} / \mathrm{A}$ carriers were available. Correlations were performed using Pearson's $r$. Data were expressed as mean \pm standard error. Two-tailed $P$-values were reported. Statistical analyses were performed using SPSS 18.0 (SPSS Inc., Chicago, IL, USA).

\section{RESULTS}

PBMCs obtained from ADA rs73598374-A carriers showed a lower TA compared to $\mathrm{G} / \mathrm{G}$ carriers $(F=8.606, P=0.019$; Figure 1a). LTL did not differ between the sexes $(F=0.563, P=0.454)$, but a significant inverse correlation with age was found $(r=-0.254, P=0.001$, Figure 2). $A D A$ rs73598374-A carriers displayed significant shorter average LTL compared to G/G carriers (1.035 \pm 0.029 vs $1.156 \pm 0.027$, $\mathrm{F}=9.096, P=0.003$, Figure $1 \mathrm{~b})$. The linear regression model confirmed the significant effect of $A D A \operatorname{rs} 73598374 \quad(\beta=-0.230$, $P=0.003)$ and age $(\beta=-0.258, P=0.001)$ on LTL, contributing to its variance for the $5.3 \%$ and $7.0 \%$, respectively. Notably, $A D A$ rs73598374-A carriers showed a stronger cross-sectional age reduction of LTL $(r=-0.314, \quad P=0.005)$ compared to $\mathrm{G} / \mathrm{G}$ carriers $(r=-0.243, P=0.022)$ (Figure 3$)$.

\section{DISCUSSION}

During the last decade several studies have demonstrated a strong link between telomere length and human lifespan. ${ }^{12}$ Telomere length shortens with age. Progressive shortening of telomeres leads to senescence, apoptosis, or oncogenic transformation of somatic cells, affecting the health and lifespan of an individual. Shorter telomeres have also been associated with increased incidence of diseases and poor survival. ${ }^{13}$

Recent evidence demonstrated a relevant role of ADA in replicative senescence through its capacity of modulating TA., ${ }^{4,5}$ Moreover, we recently reported the association between the functional $A D A$ rs73598374 and human life expectancy. 8,9

In the present work we provided first evidence for the association of $A D A$ rs73598374 with TA and LTL. In particular, carriers of ADA rs73598374-A allele showed both reduced TA and LTL compared to G/G carriers. The reduced ADA activity associated with the rs73598374-A variant predisposes those carriers to display higher levels of adenosine compared to G/G carriers. ${ }^{6,7}$ Consequently, in accordance with the fact that the chronic exposition to high levels of adenosine accelerates the process of replicative senescence, ${ }^{4} \mathrm{ADA}$ rs73598374-A carriers showed a stronger reduction of TA compared to $\mathrm{G} / \mathrm{G}$ carriers. This is in line with the results obtained on LTL measurement. Indeed, ADA rs73598374-A carriers displayed significant shorter LTL compared to G/G carriers. Notably, $A D A$ rs73598374-A carriers displayed a stronger age-dependent crosssectional decrease of LTL compared to G/G carriers, a finding in line with the association of $A D A$ rs73598374-A allele with reduced life expectancy. ${ }^{8,9}$ Interestingly, it has been demonstrated in gestional diabetes that the telomere length of fetal leukocytes is shorter than normal pregnancy, ${ }^{14}$ and that the proportion of $A D A$ rs73598374-A carrier newborns delivered by rs73598374 G/G mothers is significantly
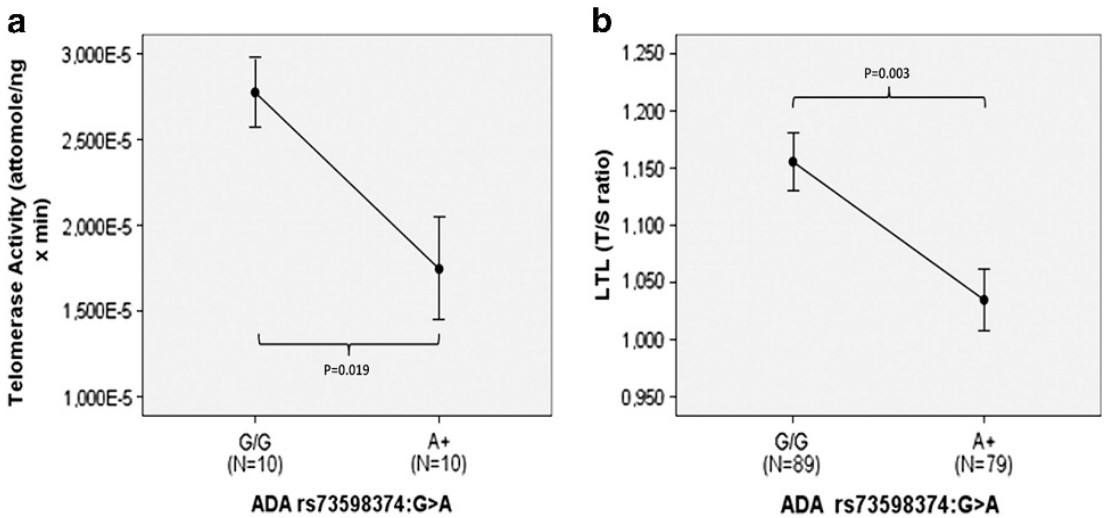

Figure 1 Effect of $A D A$ rs73598374:G>A (NM_000022.2:c.22G >A) polymorphism on TA and LTL. Graphs were obtained by general linear model using sex and age as covariates. Dots in the graphs represent the estimated marginal means for the variable under-investigation according to rs 73598374 genotypes. Vertical lines represent standard error bars around the point estimates. (a) ADA rs73598374 $\times$ TA (b) $A D A$ rs73598374 $\times$ LTL. 


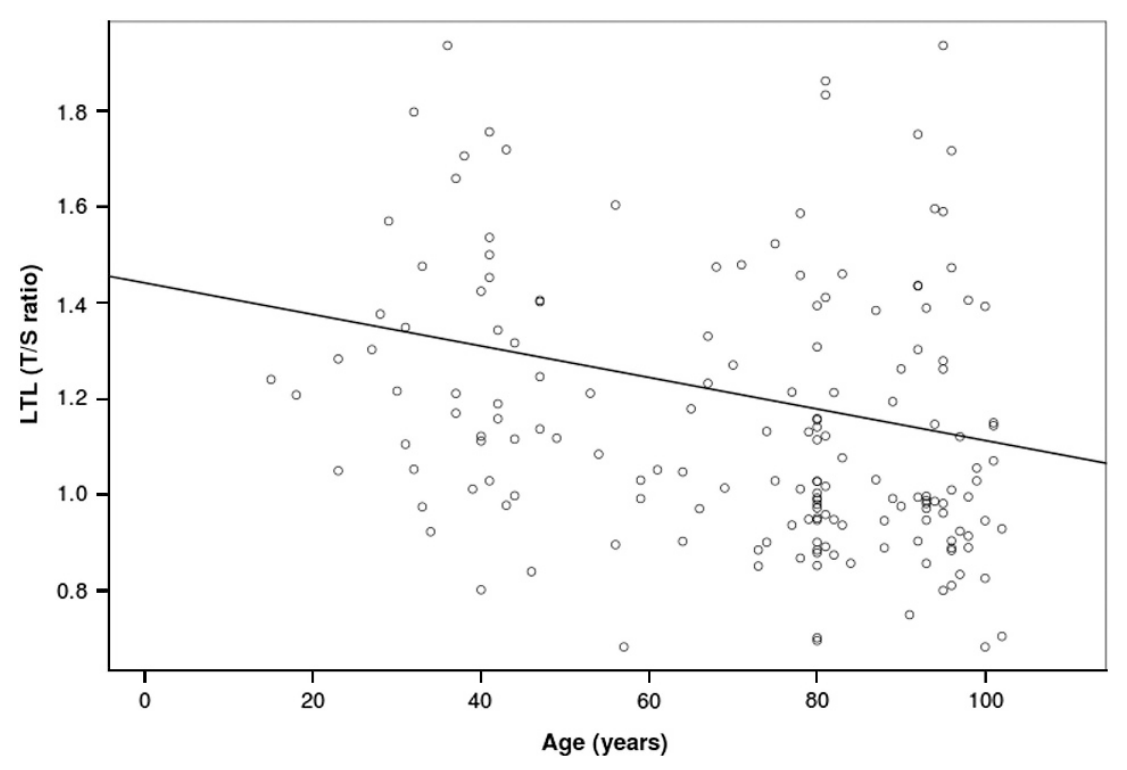

Figure 2 Correlation between age and LTL. A significant linear inverse correlation with age was observed $(r=-0.254, P=0.001)$.

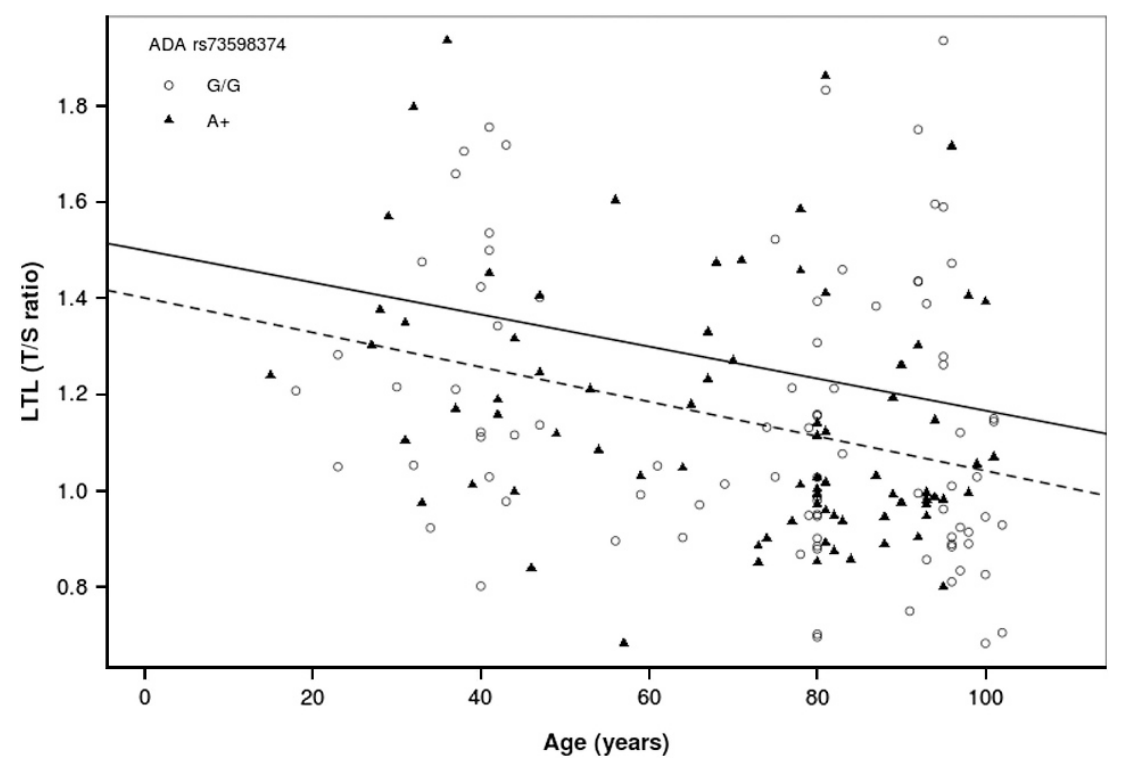

Figure 3 Cross-sectional age reduction of LTL by the ADA rs73598374:G>A (NM_000022.2:c.22G $>$ A) polymorphism. Regression lines are reported for $A D A$ rs73598374-A carriers (dashed line) and for $A D A$ rs73598374 G/G (solid line).

higher than would be expected on the basis of random mating. ${ }^{15}$ In this context, it is also remarkable that our results suggest a more prominent effect of $A D A$ rs73598374 on telomere biology at younger ages rather than later in life. Indeed, as shown in Figure 3, the regression lines are parallel, but they start at different LTL, earlier in life.

In conclusion, this is the first study reporting the association of the functional ADA rs73598374 SNP with both TA and LTL. Our study provides interesting findings that should be replicated using larger sample size (for TA especially) and detailed environmental variables (eg, smoking, working and educational status). In this context, we also believe that the association of $A D A$ rs73598374 with longevity 8,9 needs to be carefully replicated and further investigated by other research groups. The crucial role played by replicative senescence of the immune system in several human diseases (eg, cancer, autoimmune diseases and chronic infections, such as HIV) and in the aging process ${ }^{16}$ underscores the relevance of the present findings and also spurs interest into the possible involvement of $A D A$ rs73598374 SNP in shaping the susceptibility to several age-related diseases.

\section{CONFLICT OF INTEREST}

The authors declare no conflict of interest.

1 Franco R, Valenzuela A, Lluis C, Blanco J: Enzymatic and extraenzymatic role of ecto-adenosine deaminase in lymphocytes. Immunol Rev 1998; 161: 27-42.

2 Pacheco R, Martinez-Navio JM, Lejeune M et al: CD26, adenosine deaminase, and adenosine receptors mediate costimulatory signals in the immunological synapse. Proc Natl Acad Sci USA 2005; 102: 9583-9588. 
3 Butler JJ, Mader JS, Watson CL, Zhang H, Blay J, Hoskin DW: Adenosine inhibits activation-induced $\mathrm{T}$ cell expression of $\mathrm{CD} 2$ and $\mathrm{CD} 28$ co-stimulatory molecules: role of interleukin-2 and cyclic AMP signaling pathways. J Cell Biochem 2003; 89: 975-991.

4 Parish ST, Kim S, Sekhon RK, Wu JE, Kawakatsu Y, Effros RB: Adenosine deaminase modulation of telomerase activity and replicative senescence in human CD8 T lymphocytes. J Immunol 2010; 184: 2847-2854.

5 Chou JP, Ramirez CM, Wu JE, Effros RB: Accelerated aging in HIV/AIDS: novel biomarkers of senescent human CD8 + T cells. PLoS One 2013; 8: e64702.

6 Battistuzzi G, ludicone P, Santolamazza P, Petrucci R: Activity of adenosine deaminase allelic forms in intact erythrocytes and in lymphocytes. Ann Hum Genet 1981; 45: 15-19.

7 Riksen NP, Franke B, van den Broek P, Naber M, Smits P, Rongen GA: The 22G $>$ A polymorphism in the adenosine deaminase gene impairs catalytic function but does not affect reactive hyperaemia in humans in vivo. Pharmacogenet Genomics 2008; 18 : 843-846.

8 Napolioni V, Lucarini N: Gender-specific association of ADA genetic polymorphism with human longevity. Biogerontology 2010; 11: 457-462.
9 Napolioni V, Carpi FM, Giannì P et al: Age- and gender-specific epistasis between ADA and TNF- $\alpha$ influences human life-expectancy. Cytokine 2011; 56: 481-488.

10 Cawthon RM: Telomere length measurement by a novel monochrome multiplex quantitative PCR method. Nucleic Acids Res 2009; 37: e21.

11 Lan Q, Cawthon R, Gao Y et al: Longer telomere length in peripheral white blood cells is associated with risk of lung cancer and the rs2736100 (CLPTM1L-TERT) polymorphism in a prospective cohort study among women in China. PLos One 2013; 8: e59230.

12 Gomes NM, Ryder OA, Houck ML et al: Comparative biology of mammalian telomeres: hypotheses on ancestral states and the roles of telomeres in longevity determination. Aging Cell 2011; 10: 761-768.

13 Shammas MA: Telomeres, lifestyle, cancer, and aging. Curr Opin Clin Nutr Metab Care 2011; 14: 28-34.

$14 \mathrm{Xu} \mathrm{J}, \mathrm{Ye} \mathrm{J}$, Wu Y et al: Reduced fetal telomere length in gestational diabetes. PLoS One 2014; 9: e86161.

15 Borgiani P, Gloria-Bottini F, Lucarini N et al: Diabetic pregnancy: is there intrauterine selection of ADA polymorphism? Am J Hum Genet 1991; 49: 464-466.

16 Chou JP, Effros RB: T cell replicative senescence in human aging. Curr Pharm Des 2013; 19: 1680-1698. 